\title{
Fast Finite Difference Solutions of the Three Dimensional Poisson's Equation in Cylindrical Coordinates
}

\author{
Alemayehu Shiferaw, R. C. Mittal \\ Department of Mathematics, Indian Institute of Technology, Roorkee, India \\ Email: abelhaim@gmail.com,mittalrc@gmail.com
}

Received November 1, 2013; revised December 1, 2013; accepted December 8, 2013

Copyright (C) 2013 Alemayehu Shiferaw, R. C. Mittal. This is an open access article distributed under the Creative Commons Attribution License, which permits unrestricted use, distribution, and reproduction in any medium, provided the original work is properly cited.

\begin{abstract}
In this work, the three-dimensional Poisson's equation in cylindrical coordinates system with the Dirichlet's boundary conditions in a portion of a cylinder for $r \neq 0$ is solved directly, by extending the method of Hockney. The Poisson equation is approximated by second-order finite differences and the resulting large algebraic system of linear equations is treated systematically in order to get a block tri-diagonal system. The accuracy of this method is tested for some Poisson's equations with known analytical solutions and the numerical results obtained show that the method produces accurate results.
\end{abstract}

Keywords: Poisson's Equation; Hockney’s Method; Thomas Algorithm

\section{Introduction}

The three-dimensional Poisson's equation in cylindrical coordinates $(r, \theta, z)$ is given by

$$
U_{r r}+\frac{1}{r} U_{r}+\frac{1}{r^{2}} U_{\theta \theta}+U_{z z}=f(r, \theta, z)
$$

which is often encountered in heat and mass transfer theory, fluid mechanics, elasticity, electrostatics, and other areas of mechanics and physics. In particular, the Poisson equation describes stationary temperature distribution in the presence of thermal sources or sinks in the domain under consideration.

The analytic solution for the three-dimensional Poisson's equation in cylindrical coordinate system is much more complicated and tedious because of the complexity of the nature of the problems and their geometry, and the availability of appropriate methods. To solve Poisson's equation in polar and cylindrical coordinates geometry, different approaches and numerical methods using finite difference approximation have been developed. For instance, Chao [1] developed a direct solver method for the electrostatic potential in a cylindrical region; Chen [2] developed a direct spectral collocation Poisson solver for several different domains including a part of a disk, an annulus, a unit disk, and a cylinder using the weighted interpolation technique and non-classical collocation points;
Christopher [3] developed a solution method in an annulus using conformal mapping and Fast Fourier Transform; Kalita and Ray [4] have developed a high order compact scheme on a circular cylinder to solve their problem on incompressible viscous flows; Lai and Wang [5] developed a fast direct solvers for Poisson's equation on 2D polar and spherical coordinates based on FFT; Swarztrauber and Sweet [6] developed a direct solution of the discrete Poisson equation on a disk in the sense of least squares; Mittal and Gahlaut [7,8] developed high order finite difference schemes to solve Poisson's equation in cylindrical symmetry; Tan [9] developed a spectrally accurate solution for the three-dimensional Poisson's equation and Helmholtz's equation using Chebyshev series and Fourier series for a simple domain in a cylindrical coordinate system; Iyengar and Manohar [10] derived fourthorder difference schemes for the solution of the Poisson equation which occurs in problems of heat transfer; Iyengar and Goyal [11] developed a multigrid method in cylindrical coordinates system; Lai and Tseng [12] have developed a fourth-order compact scheme, and their scheme relies on the truncated Fourier series expansion, where the partial differential equations of Fourier coefficients are solved by a formally fourth-order accurate compact difference discretization; $X u$ et al. [13] developed a parallel three-dimensional Poisson solver in cylindrical co- 
ordinate system for the electrostatic potential of a charged particle beam in a circular, which used Fourier expansions in the longitudinal and azimuthal directions, and spectral element discretization in the radial direction, and some other developments had also been observed. The need to obtain the best solution for the Poisson's equation is still in progress.

In this paper, we develop a second-order finite difference approximation scheme and solve the resulting large algebraic system of linear equations systematically using block tridiagonal system [14] and extend the Hockney's method [15] to solve the three dimensional Poisson's equation on Cylindrical coordinates system.

\section{Finite Difference Approximation}

Consider the three dimensional Poisson's equation in cylindrical coordinates $(r, \theta, z)$ given by

$$
\frac{\partial^{2} U}{\partial r^{2}}+\frac{1}{r} \frac{\partial U}{\partial r}+\frac{1}{r^{2}} \frac{\partial^{2} U}{\partial \theta^{2}}+\frac{\partial^{2} U}{\partial z^{2}}=f(r, \theta, z) \text { on } D
$$

and the boundary condition

$$
U(r, \theta, z)=g(r, \theta, z) \text { on } C
$$

where $C$ is the boundary of $D$ and $D$ is

$$
\begin{aligned}
D_{1}=\{( & (r, \theta, z): R_{0}<r<R_{1}, a<z<b, \\
& \left.\theta_{0}<\theta<\theta_{1}, \theta_{0}<\theta_{1}<2 \pi\right\}
\end{aligned}
$$

and

$$
D_{2}=\left\{(r, \theta, z): R_{0}<r<R_{1}, a<z<b, 0 \leq \theta<2 \pi\right\}
$$

Consider Figure 1 as the geometry of the problem. Let $u(r, \theta, z)$ be discretized at the point $\left(r_{i}, \theta_{j}, z_{k}\right)$ and for simplicity write a point $\left(r_{i}, \theta_{j}, z_{k}\right)$ as $(i, j, k)$ and $u\left(r_{i}, \theta_{j}, z_{k}\right)$ as $u_{i, j, k}$.

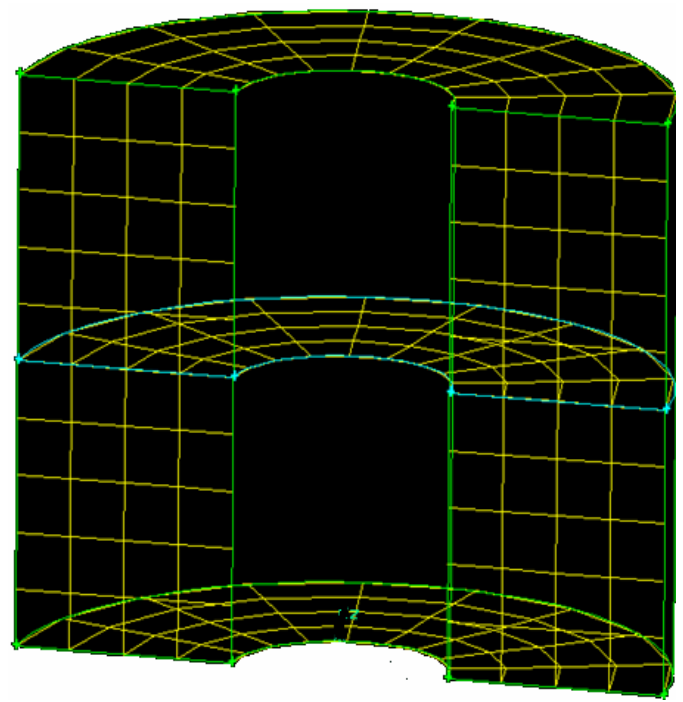

Figure 1. Portion of a Cylinder.
Assume that there are $\mathrm{M}$ points along $r, \mathrm{~N}$ points along $\theta$ and $\mathrm{P}$ points along the $\mathrm{Z}$ directions to form the mesh, and let the step size along the direction of $r$ be $\Delta r$, of $\theta$ be $\Delta \theta$ and $z$ be $\Delta z$.

Here $r_{i}=R_{0}+i \Delta r, \theta_{j}=\theta_{0}+j \Delta \theta$ and $z_{k}=a+k \Delta z$ where $i=1,2, \cdots, M, j=1,2, \cdots, N$ and $k=1,2, \cdots, P$ For $r \neq 0$, using the central difference approximation scheme that

$$
\begin{aligned}
& \frac{\partial u}{\partial r}=\frac{u_{i+1, j, k}-u_{i-1, j, k}}{2 \Delta r}+O(\Delta r) \\
& \frac{\partial^{2} u}{\partial r^{2}}=\frac{u_{i+1, j, k}-2 u_{i, j, k}+u_{i-1, j, k}}{(\Delta r)^{2}}+O\left((\Delta r)^{2}\right) \\
& \frac{\partial^{2} u}{\partial \theta^{2}}=\frac{u_{i, j+1, k}-2 u_{i, j, k}+u_{i, j-1, k}}{(\Delta \theta)^{2}}+O\left((\Delta \theta)^{2}\right) \text { and } \\
& \frac{\partial^{2} u}{\partial z^{2}}=\frac{u_{i, j, k+1}-2 u_{i, j, k}+u_{i, j, k-1}}{(\Delta z)^{2}}+O\left((\Delta z)^{2}\right)
\end{aligned}
$$

Truncating higher order differences of (3) and substituting (3) in (2), we have

$$
\begin{aligned}
& f_{i, j, k}=\frac{u_{i+1, j, k}-2 u_{i, j, k}+u_{i-1, j, k}}{(\Delta r)^{2}}+\frac{u_{i+1, j, k}-u_{i-1, j, k}}{(2 \Delta r) r_{i}} \\
& +\frac{1}{r_{i}^{2}}\left(\frac{u_{i, j+1, k}-2 u_{i, j, k}+u_{i, j-1, k}}{(\Delta \theta)^{2}}\right)+\frac{u_{i, j, k+1}-2 u_{i, j, k}+u_{i, j, k-1}}{(\Delta z)^{2}}
\end{aligned}
$$

Let $\omega_{i}=\frac{\Delta r}{2 r_{i}}, \quad \alpha_{i}=\frac{(\Delta r)^{2}}{r_{i}^{2}(\Delta \theta)^{2}}, \rho=\frac{(\Delta r)^{2}}{(\Delta z)^{2}} \quad$ and $y_{i}=-2\left(1+\alpha_{i}+\rho\right)$.

Multiplying both sides of (4) by $(\Delta r)^{2}$, rearranging and simplifying further, we get

$$
\begin{aligned}
& (\Delta r)^{2} f_{i, j, k}=\left(1+\omega_{i}\right) u_{i+1, j, k}+\left(1-\omega_{i}\right) u_{i-1, j, k} \\
& +\alpha_{i}\left(u_{i, j+1, k}+u_{i, j-1, k}\right)+\rho\left(u_{i, j, k+1}+u_{i, j, k-1}\right)+y_{i} u_{i, j, k}
\end{aligned}
$$

When there are two or more space dimensions the band width is larger and the number of operations goes up and thus the computation for the solution is not such an easy task.

The system of Equations in (5) is a linear sparse system, and thereby saving on both work and storage compared with a general system of equations. Such savings are basically true of finite difference methods: they yield sparse systems because each equation involves only a few variables. Now we use these advantages.

Consider Equation (5) first in the $\theta$ direction, next in the $z$ direction and lastly in the $r$ direction, and hence Equation (5) can be put in matrix form as

$$
A \boldsymbol{U}=\mathcal{B}
$$


where $A=\left(\begin{array}{ccccccccc}R_{1} & S_{1} & & & & & & \\ S_{2}^{*} & R_{2} & S_{2} & & & & & \\ & S_{3}^{*} & R_{3} & S_{3} & & & & \\ & & & & \ddots & & & \\ & & & & & S_{M-1}^{*} & R_{M-1} & S_{M-1} \\ & & & & & & S_{M}^{*} & R_{M}\end{array}\right)$

it has $M$ blocks and each block is of order NP.

$$
R_{i}=\left(\begin{array}{ccccccccc}
L_{i} & \mathrm{~T} & & & & & & \\
\mathrm{~T} & L_{i} & \mathrm{~T} & & & & & \\
& \mathrm{~T} & L_{i} & \mathrm{~T} & & & & \\
& & & & \ddots & & & \\
& & & & & \mathrm{T} & L_{i} & \mathrm{~T} \\
& & & & & & \mathrm{~T} & L_{i}
\end{array}\right)
$$

For $D_{1}$,

$$
L_{i}=\left(\begin{array}{cccccccc}
y_{i} & \alpha_{i} & & & & & & \\
\alpha_{i} & y_{i} & \alpha_{i} & & & & & \\
& \alpha_{i} & y_{i} & \alpha_{i} & & & & \\
& & & & \ddots & & & \\
& & & & & \alpha_{i} & y_{i} & \alpha_{i} \\
& & & & & & \alpha_{i} & y_{i}
\end{array}\right)
$$

For $D_{2}$,

$$
L_{i}=\left(\begin{array}{cccccccc}
y_{i} & \alpha_{i} & & & & & & \alpha_{i} \\
\alpha_{i} & y_{i} & \alpha_{i} & & & & & \\
& \alpha_{i} & y_{i} & \alpha_{i} & & & & \\
& & & & \ddots & & & \\
& & & & & \alpha_{i} & y_{i} & \alpha_{i} \\
\alpha_{i} & & & & & & \alpha_{i} & y_{i}
\end{array}\right)
$$

is a circulant matrix;

Both matrices (8) and (9) are of order $\mathrm{N}$; and $\mathrm{T}=\operatorname{diag}(\rho, \rho, \rho, \cdots, \rho)$ is of order $\mathrm{N}$.

$$
S_{i}=\operatorname{diag}\left(\omega_{i}, \omega_{i}, \omega_{i}, \cdots, \omega_{i}\right) \text { has P blocks and }
$$

$\boldsymbol{\omega}_{i}=\operatorname{diag}\left(1+\omega_{i}, 1+\omega_{i}, \cdots, 1+\omega_{i}\right)$ is of order $\mathrm{N}$

$S_{i}^{*}=\operatorname{diag}\left(\boldsymbol{\varphi}_{i}, \boldsymbol{\varphi}_{i}, \boldsymbol{\varphi}_{i}, \cdots, \boldsymbol{\varphi}_{i}\right)$ has P blocks and

$\boldsymbol{\varphi}_{i}=\operatorname{diag}\left(1-\omega_{i}, 1-\omega_{i}, \cdots, 1-\omega_{i}\right)$ is of order $\mathrm{N}$.

$$
\mathcal{B}=\left[\begin{array}{lllll}
\boldsymbol{B}_{1} & \boldsymbol{B}_{2} & \boldsymbol{B}_{3} & \cdots & \boldsymbol{B}_{M}
\end{array}\right]^{\mathrm{T}},
$$

$\boldsymbol{B}_{k}=\left[\begin{array}{llll}\boldsymbol{d}_{1 k} & \boldsymbol{d}_{2 k} & \cdots & \boldsymbol{d}_{N k}\end{array}\right]^{\mathrm{T}}$ and

$\boldsymbol{d}_{j k}=\left[\begin{array}{llll}d_{1 j k} & d_{2 j k} & \cdots & d_{M j k}\end{array}\right]^{\mathrm{T}}$ such that each $d_{i j k}$ represents a known boundary values of $U$ and values of $f$, and $\boldsymbol{U}=\left[\begin{array}{lllll}\boldsymbol{U}_{1} & \boldsymbol{U}_{2} & \boldsymbol{U}_{3} & \cdots & \boldsymbol{U}_{M}\end{array}\right]^{\mathrm{T}}$,

$\boldsymbol{U}_{i}=\left(\begin{array}{lllll}U_{i 1} & U_{i 2} & U_{i 3} & \cdots & U_{i P}\end{array}\right)^{\mathrm{T}}$ and

$U_{i k}=\left(\begin{array}{lllll}U_{i j 1} & U_{i j 2} & U_{i j 3} & \cdots & U_{i j P}\end{array}\right)^{\mathrm{T}}$

We write (6) a

$$
\begin{aligned}
& R_{1} \boldsymbol{U}_{1}+S_{1} \boldsymbol{U}_{2}=\boldsymbol{B}_{1} \\
& S_{2}^{*} \boldsymbol{U}_{1}+R_{2} \boldsymbol{U}_{2}+S_{2} \boldsymbol{U}_{3}=\boldsymbol{B}_{2} \\
& S_{3}^{*} \boldsymbol{U}_{2}+R_{3} \boldsymbol{U}_{3}+S_{3} \boldsymbol{U}_{4}=\boldsymbol{B}_{3} \\
& \quad \vdots \\
& S_{M}^{*} \boldsymbol{U}_{M-1}+R_{M} \boldsymbol{U}_{M}=\boldsymbol{B}_{M}
\end{aligned}
$$

\section{Extended Hockney's Method}

Observe that the matrix $L_{i}$ is a real symmetric matrix and hence its eigenvalues and eigenvectors can easily be obtained.

for $D_{1} \quad \lambda_{i j}=y_{i}+2 \alpha_{i} \cos \left(\frac{j \pi}{N+1}\right)$ and

for $D_{2} \quad \lambda_{i j}=y_{i}+2 \alpha_{i} \cos \left(\frac{2 \pi j}{N}\right) \quad i=1,2,3, \cdots, M$,

$j=1,2,3, \cdots, N$.

Let $\boldsymbol{q}_{j}$ be an eigenvector of $L_{i}$ corresponding to the eigenvalue $\lambda_{i j}$ and the matrix

$Q=\left[\begin{array}{lllll}\boldsymbol{q}_{1} & \boldsymbol{q}_{2} & \boldsymbol{q}_{3} & \cdots & \boldsymbol{q}_{n}\end{array}\right]^{\mathrm{T}}$ be a modal matrix of $L_{i}$,

$\forall i$ such that $Q Q^{\mathrm{T}}=I$ and

$Q^{\mathrm{T}} L_{i} Q=\operatorname{diag}\left(\eta_{i 1}, \eta_{i 2}, \eta_{i 3}, \cdots, \eta_{i N}\right)$

The $N \times N$ modal matrix $\mathrm{Q}$ is defined by

$q_{i, j}=\sqrt{\frac{2}{N+1}} \sin \left(\frac{i j \pi}{N+1}\right)$ for $D_{1}$ and

$q_{i, j}=\frac{(\cos \theta+\sin \theta)}{\sqrt{N}}$, where $\theta=\left(\frac{2 \pi}{N}\right)(i-1)(j-1)$ for

$D_{2}$ and $i=1,2,3, \cdots, M, j=1,2,3, \cdots, N$

Let $\mathbb{Q}=\operatorname{diag}(Q, Q, \cdots, Q)$ be a matrix of order NP.

Thus $\mathbb{Q}$ satisfy the property that $\mathbb{Q}^{\mathrm{T}} \mathbb{Q}=I$ since $Q^{\mathrm{T}} Q=I$ and due to the matrix $R_{i}$ is symmetric, we have $\mathbb{Q}^{\mathrm{T}} R_{i} \mathbb{Q}=\operatorname{diag}\left(\eta_{j 1}^{i}, \eta_{j 2}^{i}, \cdots, \eta_{j \mathrm{P}}^{i}\right)=\Upsilon_{i}$ (say);

$\mathbb{Q}^{\mathrm{T}} S_{i} \mathbb{Q}=S_{i}$ and $\mathbb{Q}^{\mathrm{T}} S_{i}^{*} \mathbb{Q}=S_{i}^{*}$ since both $S_{i}$ and $S_{i}^{*}$ are diagonal matrices.

Let $\mathbb{Q}^{\mathrm{T}} \boldsymbol{U}_{i}=\boldsymbol{V}_{i} \Rightarrow \boldsymbol{U}_{i}=\mathbb{Q} \boldsymbol{V}_{i}$

$$
\mathbb{Q}^{\mathrm{T}} \boldsymbol{B}_{i}=\overline{\boldsymbol{b}}_{i} \Rightarrow \boldsymbol{B}_{i}=\mathbb{Q} \overline{\boldsymbol{b}}_{i}
$$

where $\boldsymbol{V}_{i}=\left[\begin{array}{lllll}V_{i 1} & V_{i 2} & V_{i 3} & \cdots & V_{i P}\end{array}\right]^{\mathrm{T}}$,

$$
\begin{aligned}
& V_{i k}=\left[\begin{array}{lllll}
v_{i 1 k} & v_{i 2 k} & v_{i 3 k} & \cdots & v_{i N k}
\end{array}\right]^{\mathrm{T}} ; \\
& \overline{\boldsymbol{b}}_{i}=\left[\begin{array}{llll}
\boldsymbol{b}_{i 1} & \boldsymbol{b}_{i 2 ?} & \cdots & \boldsymbol{b}_{i k}
\end{array}\right]^{\mathrm{T}} \text { and } \\
& \boldsymbol{b}_{i k}=\left[\begin{array}{llll}
b_{i 1 k} & b_{i 2 k} & \cdots & b_{i N k}
\end{array}\right]^{\mathrm{T}}
\end{aligned}
$$

Pre-multiplying Equation (10) by $\mathbb{Q}^{\mathrm{T}}$ and applying (11), we get

$$
\begin{gathered}
\Upsilon_{1} \boldsymbol{V}_{1}+S_{1} \boldsymbol{V}_{2}=\overline{\boldsymbol{b}}_{1} \\
S_{2}^{*} \boldsymbol{V}_{1}+\Upsilon_{2} \boldsymbol{V}_{2}+S_{2} \boldsymbol{V}_{3}=\overline{\boldsymbol{b}}_{2} \\
S_{3}^{*} \boldsymbol{V}_{2}+\Upsilon_{3} \boldsymbol{V}_{3}+S_{3} \boldsymbol{V}_{4}=\overline{\boldsymbol{b}}_{3} \\
\vdots \\
S_{M}^{*} \boldsymbol{V}_{M-1}+\Upsilon_{M} \boldsymbol{V}_{M}=\overline{\boldsymbol{b}}_{M}
\end{gathered}
$$


Now from each Equation of (14) we collect the first equations and put them as one group of equation

$$
\begin{aligned}
& \eta_{j k}^{1} V_{k}^{1}+\left(1+\omega_{1}\right) V_{k}^{2}=\overline{\boldsymbol{b}}_{1} \\
& \left(1-\omega_{2}\right) V_{k}^{1}+\eta_{j k}^{2} V_{k}^{2}+\left(1+\omega_{2}\right) V_{k}^{3}=\overline{\boldsymbol{b}}_{2} \\
& \left(1-\omega_{2}\right) V_{k}^{2}+\eta_{j k}^{3} V_{k}^{3}+\left(1+\omega_{2}\right) V_{k}^{4}=\overline{\boldsymbol{b}}_{3} \\
& \quad \vdots \\
& \left(1-\omega_{M-1}\right) V_{k}^{M-2}+\eta_{j k}^{M} V_{k}^{M-1}+\left(1+\omega_{M-1}\right) V_{k}^{M}=\overline{\boldsymbol{b}}_{M-1} \\
& \left(1-\omega_{M}\right) V_{k}^{M-1}+\eta_{j k}^{M} V_{k}^{M}=\overline{\boldsymbol{b}}_{M}
\end{aligned}
$$

Collect as the first set of equations by putting $k=1$ in Equation (15), for $i=1,2,3, \cdots, M$ and $j=1,2,3, \cdots, N$ $\left(1-\omega_{i}\right) v_{j 1}^{i-1}+\eta_{j 1}^{i} v_{j 1}^{i}+\left(1+\omega_{i}\right) v_{j 1}^{i+1}=b_{j 1}^{i}$ and

$$
v_{j 1}^{0}=0=v_{j 1}^{m}
$$

Again consider the second equations by putting $k=2$, and get

$$
\begin{gathered}
\left(1-\omega_{i}\right) v_{j 2}^{i-1}+\eta_{j 2}^{i} v_{j 2}^{i}+\left(1+\omega_{i}\right) v_{j 2}^{i+1}=b_{j 2}^{i} \quad \text { and } \\
v_{j 2}^{0}=0=v_{j 2}^{m}
\end{gathered}
$$

Continuing in this manner and finally considering the last equations for $k=P$, we obtain

$$
\begin{gathered}
\left(1-\omega_{i}\right) v_{j P}^{i-1}+\eta_{j 2}^{i} v_{j P}^{i}+\left(1+\omega_{i}\right) v_{j P}^{i+1}=b_{j P}^{i} \quad \text { and } \\
v_{j P}^{0}=0=v_{j P}^{m}
\end{gathered}
$$

All these set of Equations (14a)-(14c) are tri-diagonal ones and hence we solve for $v_{j k}^{i}$ by using Thomas algorithm. With the help of (11) again we get all $u_{j k}^{i}$ and this solves (5) as desired. By doing this we generally reduce the number of computations and computational time.

\section{Numerical Results}

In order to test the efficiency and adaptability of the proposed method, computational experiments are done on some selected problems that may arise in practice, for which the analytical solutions of $U$ are known to us. The computed solutions are found for any values of $\mathrm{M}, \mathrm{N}$, and P. Here results are reported at some randomly taken points from Tables 1 to 7.

Example 1. Consider $\nabla^{2} U=-3 \cos \theta$ with the boundary conditions

$$
\begin{gathered}
U(0, \theta, z)=U(1, \theta, z)=-2 z \\
U(r, 0, z)=r(1-r)-2 z, \quad U\left(r, \frac{\pi}{2}, z\right)=-2 z \\
(r, \theta, 0)=r(1-r) \cos \theta, \quad U(r, \theta, 1)=r(1-r) \cos \theta-2
\end{gathered}
$$

The analytical solution is $U(r, \theta, z)=r(1-r) \cos \theta-2 z$ and the results of this example are shown in Table $\mathbf{1}$.

Example 2. Consider $\nabla^{2} U=-\pi^{2} r \cos \theta \sin (\pi z)$ with the boundary conditions

$$
\begin{gathered}
U(1, \theta, z)=\cos \theta \sin (\pi z), U(2, \theta, z)=2 \cos \theta \sin (\pi z) \\
U(r, 0, z)=r \sin (\pi z), U(r, \pi, z)=-r \sin (\pi z) \\
U(r, \theta, 0)=U(r, \theta, 1)=0
\end{gathered}
$$

The analytical solution is $U(r, \theta, z)=r \cos \theta \sin (\pi z)$ and results of this example are shown in Table 2

Example 3. Consider

$$
\nabla^{2} U=-\pi^{2}\left(r^{2}-\frac{1}{r^{2}}\right) \sin (2 \theta) \sin (\pi z)
$$

with the boundary conditions

$$
U(1, \theta, z)=0, \quad U(2, \theta, z)=\frac{15}{4} \sin (2 \theta) \sin (\pi z)
$$

$U(r, 0, z)=0=U\left(r, \frac{\pi}{2}, z\right)$ and

$U(r, \theta, 0)=0=U(r, \theta, 1)$

The analytical solution is

Table 1. Maximum absolute error of example 1.

\begin{tabular}{cccc}
\hline$(N, P, M)$ & Max. absolute error & $(N, P, M)$ & Max. absolute error \\
\hline$(9,9,9)$ & $6.83901 \mathrm{e}-005$ & $(29,9,39)$ & $7.63105 \mathrm{e}-006$ \\
$(9,9,29)$ & $6.85359 \mathrm{e}-005$ & $(29,19,9)$ & $7.64055 \mathrm{e}-006$ \\
$(9,19,9)$ & $6.85882 \mathrm{e}-005$ & $(29,29,19)$ & $7.63684 \mathrm{e}-006$ \\
$(9,19,19)$ & $6.85881 \mathrm{e}-005$ & $(29,29,29)$ & $7.65726 \mathrm{e}-006$ \\
$(9,29,39)$ & $6.87927 \mathrm{e}-005$ & $(29,39,19)$ & $7.63846 \mathrm{e}-006$ \\
$(9,39,29)$ & $6.88027 \mathrm{e}-005$ & $(29,39,29)$ & $7.65884 \mathrm{e}-006$ \\
$(19,9,9)$ & $1.71132 \mathrm{e}-005$ & $(39,9,19)$ & $4.28351 \mathrm{e}-006$ \\
$(19,9,19)$ & $1.71127 \mathrm{e}-005$ & $(39,9,39)$ & $4.29603 \mathrm{e}-006$ \\
$(19,19,19)$ & $1.71675 \mathrm{e}-005$ & $(39,19,29)$ & $4.30845 \mathrm{e}-006$ \\
$(19,29,39)$ & $1.72164 \mathrm{e}-005$ & $(39,29,19)$ & $4.29998 \mathrm{e}-006$ \\
$(19,39,9)$ & $1.71752 \mathrm{e}-005$ & $(39,39,9)$ & $4.30369 \mathrm{e}-006$ \\
$(19,39,39)$ & $1.72198 \mathrm{e}-005$ & $(39,39,39)$ & $4.31261 \mathrm{e}-006$ \\
\hline
\end{tabular}

Table 2. Maximum absolute error of example 2.

\begin{tabular}{cccc}
\hline$(N, P, M)$ & Max. absolute error & $(N, P, M)$ & Max. absolute error \\
\hline$(9,9,9)$ & $5.97565 \mathrm{e}-003$ & $(29,9,39)$ & $6.25865 \mathrm{e}-003$ \\
$(9,9,29)$ & $6.04232 \mathrm{e}-003$ & $(29,19,9)$ & $1.56933 \mathrm{e}-003$ \\
$(9,19,9)$ & $1.68198 \mathrm{e}-003$ & $(29,29,19)$ & $7.21066 \mathrm{e}-004$ \\
$(9,19,19)$ & $1.69672 \mathrm{e}-003$ & $(29,29,29)$ & $7.21498 \mathrm{e}-004$ \\
$(9,29,39)$ & $8.9486 \mathrm{e}-004$ & $(29,39,19)$ & $4.18664 \mathrm{e}-004$ \\
$(9,39,29)$ & $6.14273 \mathrm{e}-004$ & $(29,39,29)$ & $4.19068 \mathrm{e}-004$ \\
$(19,9,9)$ & $6.18349 \mathrm{e}-003$ & $(39,9,19)$ & $6.23836 \mathrm{e}-003$ \\
$(19,9,19)$ & $6.24022 \mathrm{e}-003$ & $(39,9,39)$ & $6.24401 \mathrm{e}-003$ \\
$(19,19,19)$ & $1.6095 \mathrm{e}-003$ & $(39,19,29)$ & $1.5717 \mathrm{e}-003$ \\
$(19,29,39)$ & $7.53504 \mathrm{e}-004$ & $(39,29,19)$ & $7.07837 \mathrm{e}-004$ \\
$(19,39,9)$ & $4.50526 \mathrm{e}-004$ & $(39,39,9)$ & $4.01493 \mathrm{e}-004$ \\
$(19,39,39)$ & $4.53562 \mathrm{e}-004$ & $(39,39,39)$ & $4.05867 \mathrm{e}-004$ \\
\hline
\end{tabular}


$U=\left(r^{2}-\frac{1}{r^{2}}\right) \sin (2 \theta) \sin (\pi z)$ and results of this example are shown in Table 3

\section{Example 4. Consider}

$$
\begin{aligned}
& \nabla^{2} U=\left(\cos \theta_{1}+\sin \theta_{1}\right)\left(\cos z_{1}+\sin z_{1}\right) \\
& \times\left(\left(\frac{\pi}{2 r}-\frac{\pi^{2}}{2}-4 \frac{\pi^{2}}{r^{2}}\right) \cos r^{*}-\left(\frac{\pi}{2 r}+\frac{\pi^{2}}{2}+4 \frac{\pi^{2}}{r^{2}}\right) \sin r^{*}\right)
\end{aligned}
$$

where $\quad r^{*}=\frac{\pi}{2}(r-4), \quad \theta_{1}=\pi(2 \theta-1), \quad z_{1}=\frac{\pi}{2}(z-1)$, $2 \leq r \leq 4,0 \leq \theta \leq 0.5$ and $-1 \leq z \leq 1$

The analytical solution is

$$
\begin{aligned}
U(r, \theta, z)= & \left(\cos r^{*}+\sin r^{*}\right)\left(\cos \theta_{1}+\sin \theta_{1}\right) \\
& \times\left(\cos z_{1}+\sin z_{1}\right)
\end{aligned}
$$

This problem was considered as one test problem by Iyengar and Goyal [11] and their result and our are found to be the same for $h=1 / 8$, but their method cannot be applied for non-uniform values $h_{1}, h_{2}$ and $h_{3}$. We have shown the results of this example in Table 4.

Table 3. Maximum absolute error of example 3.

\begin{tabular}{cccc}
\hline$(N, P, M)$ & Max. absolute error & $(N, P, M)$ & Max. absolute error \\
\hline$(9,9,9)$ & $1.0041 \mathrm{e}-002$ & $(29,9,39)$ & $9.14135 \mathrm{e}-003$ \\
$(9,9,29)$ & $1.03659 \mathrm{e}-002$ & $(29,19,9)$ & $2.09239 \mathrm{e}-003$ \\
$(9,19,9)$ & $3.33415 \mathrm{e}-003$ & $(29,29,19)$ & $1.08113 \mathrm{e}-003$ \\
$(9,19,19)$ & $3.5776 \mathrm{e}-003$ & $(29,29,29)$ & $1.12241 \mathrm{e}-003$ \\
$(9,29,39)$ & $2.39279 \mathrm{e}-003$ & $(29,39,19)$ & $6.45366 \mathrm{e}-004$ \\
$(9,39,29)$ & $1.94206 \mathrm{e}-003$ & $(29,39,29)$ & $6.85093 \mathrm{e}-004$ \\
$(19,9,9)$ & $8.97807 \mathrm{e}-003$ & $(39,9,19)$ & $8.98428 \mathrm{e}-003$ \\
$(19,9,19)$ & $9.24935 \mathrm{e}-003$ & $(39,9,39)$ & $9.07348 \mathrm{e}-003$ \\
$(19,19,19)$ & $2.51841 \mathrm{e}-003$ & $(39,19,29)$ & $2.30437 \mathrm{e}-003$ \\
$(19,29,39)$ & $1.33191 \mathrm{e}-003$ & $(39,29,19)$ & $1.01451 \mathrm{e}-003$ \\
$(19,39,9)$ & $6.40825 \mathrm{e}-004$ & $(39,39,9)$ & $3.98681 \mathrm{e}-004$ \\
$(19,39,39)$ & $8.95344 \mathrm{e}-004$ & $(39,39,39)$ & $6.31752 \mathrm{e}-004$ \\
\hline
\end{tabular}

Table 4. Maximum absolute error of example 4.

\begin{tabular}{cccc}
\hline$(N, P, M)$ & Max. absolute error & $(N, P, M)$ & Max. absolute error \\
\hline$(9,9,9)$ & $1.52153 \mathrm{e}-002$ & $(29,9,39)$ & $4.59667 \mathrm{e}-003$ \\
$(9,9,29)$ & $1.18196 \mathrm{e}-002$ & $(29,19,9)$ & $5.9446 \mathrm{e}-003$ \\
$(9,19,9)$ & $1.26939 \mathrm{e}-002$ & $(29,29,19)$ & $2.29605 \mathrm{e}-003$ \\
$(9,19,19)$ & $9.82575 \mathrm{e}-003$ & $(29,29,29)$ & $1.72475 \mathrm{e}-003$ \\
$(9,29,39)$ & $8.67411 \mathrm{e}-003$ & $(29,39,19)$ & $2.13081 \mathrm{e}-003$ \\
$(9,39,29)$ & $8.68314 \mathrm{e}-003$ & $(29,39,29)$ & $1.558 \mathrm{e}-003$ \\
$(19,9,9)$ & $9.48954 \mathrm{e}-003$ & $(39,9,19)$ & $4.98915 \mathrm{e}-003$ \\
$(19,9,19)$ & $6.4572 \mathrm{e}-003$ & $(39,9,39)$ & $4.21468 \mathrm{e}-003$ \\
$(19,19,19)$ & $3.87647 \mathrm{e}-003$ & $(39,19,29)$ & $1.82246 \mathrm{e}-003$ \\
$(19,29,39)$ & $2.66641 \mathrm{e}-003$ & $(39,29,19)$ & $1.91856 \mathrm{e}-003$ \\
$(19,39,9)$ & $6.34865 \mathrm{e}-003$ & $(39,39,9)$ & $4.97913 \mathrm{e}-003$ \\
$(19,39,39)$ & $2.49938 \mathrm{e}-003$ & $(39,39,39)$ & $9.7357 \mathrm{e}-004$ \\
\hline
\end{tabular}

Example 5. Consider $\nabla^{2} U=-3 \cos \theta$ with the boundary conditions

$$
\begin{gathered}
U(0, \theta, z)=z=U(1, \theta, z), \\
U(r, \theta, 0)=r(1-r) \cos \theta, U(r, \theta, 1)=1+r(1-r) \cos \theta
\end{gathered}
$$

The analytical solution is $U=r(1-r) \cos \theta+z$ and results of this example are shown in Table 5.

Example 6. Consider $\nabla^{2} U=6 r z \cos \theta$ with the boundary conditions

$$
\begin{gathered}
U(0, \theta, z)=0, U(1, \theta, z)=z \cos ^{3} \theta, \\
U(r, \theta, 0)=0 \text { and }(r, \theta, 1)=r^{3} \cos ^{3} \theta
\end{gathered}
$$

The analytical solution is $U=r^{3} z \cos ^{3} \theta$ and results of this example are shown in Table 6.

Example 7. Consider

$$
\nabla^{2} U=-\pi^{2}\left(r^{2}-1 / r^{2}\right) \sin (2 \theta) \sin (\pi z)
$$

with the boundary conditions

$$
\begin{gathered}
U(1, \theta, z)=0, U(2, \theta, z)=\frac{15}{4} \sin (2 \theta) \sin (\pi z) \\
U(r, \theta, 0)=0=U(r, \theta, 1)
\end{gathered}
$$

The analytical solution is $U=\left(r^{2}-\frac{1}{r^{2}}\right) \sin (2 \theta) \sin (\pi z)$ and results of this example are shown in Table 7. Here, we have displayed only for some points which are taken randomly, but we can show the results at any point inside the cylinder.

\section{Conclusion}

In this work, first we apply Hockney's method in order to reduce (5) as a tri-diagonal matrix and after that all the computations directly rely on the Thomas Algorithm. By

Table 5. Maximum absolute error of example 5.

\begin{tabular}{cccc}
\hline$(N, P, M)$ & Max. absolute error & $(N, P, M)$ & Max. absolute error \\
\hline$(10,9,9)$ & $2.91054 \mathrm{e}-003$ & $(29,9,39)$ & $3.27118 \mathrm{e}-004$ \\
$(10,9,29)$ & $2.94732 \mathrm{e}-003$ & $(29,19,9)$ & $3.2355 \mathrm{e}-004$ \\
$(10,19,9)$ & $2.91942 \mathrm{e}-003$ & $(29,29,19)$ & $3.26362 \mathrm{e}-004$ \\
$(10,19,19)$ & $2.94503 \mathrm{e}-003$ & $(29,29,29)$ & $3.27939 \mathrm{e}-004$ \\
$(10,29,39)$ & $2.96297 \mathrm{e}-003$ & $(29,39,19)$ & $3.26426 \mathrm{e}-004$ \\
$(10,39,29)$ & $2.95912 \mathrm{e}-003$ & $(29,39,29)$ & $3.28008 \mathrm{e}-004$ \\
$(20,9,9)$ & $7.26075 \mathrm{e}-004$ & $(39,9,19)$ & $1.82891 \mathrm{e}-004$ \\
$(20,9,19)$ & $7.32065 \mathrm{e}-004$ & $(39,9,39)$ & $1.83977 \mathrm{e}-004$ \\
$(20,19,19)$ & $7.34274 \mathrm{e}-004$ & $(39,19,29)$ & $1.84332 \mathrm{e}-004$ \\
$(20,29,39)$ & $7.39078 \mathrm{e}-004$ & $(39,29,19)$ & $1.83546 \mathrm{e}-004$ \\
$(20,39,9)$ & $7.28843 \mathrm{e}-004$ & $(39,39,9)$ & $1.8211 \mathrm{e}-004$ \\
$(20,39,39)$ & $7.3923 \mathrm{e}-004$ & $(39,39,39)$ & $1.84702 \mathrm{e}-004$ \\
\hline
\end{tabular}


Table 6. Maximum absolute error of example 6.

\begin{tabular}{cccc}
\hline$(N, P, M)$ & Max. absolute error & $(N, P, M)$ & Max. absolute error \\
\hline$(10,9,9)$ & $8.08303 \mathrm{e}-003$ & $(29,9,39)$ & $8.96012 \mathrm{e}-004$ \\
$(10,9,29)$ & $7.78161 \mathrm{e}-003$ & $(29,19,9)$ & $1.26723 \mathrm{e}-003$ \\
$(10,19,9)$ & $8.13051 \mathrm{e}-003$ & $(29,29,19)$ & $9.73704 \mathrm{e}-004$ \\
$(10,19,19)$ & $7.87559 \mathrm{e}-003$ & $(29,29,29)$ & $9.19304 \mathrm{e}-004$ \\
$(10,29,39)$ & $7.82057 \mathrm{e}-003$ & $(29,39,19)$ & $9.74094 \mathrm{e}-004$ \\
$(10,39,29)$ & $7.84024 \mathrm{e}-003$ & $(29,39,29)$ & $9.19684 \mathrm{e}-004$ \\
$(20,9,9)$ & $2.33516 \mathrm{e}-003$ & $(39,9,19)$ & $5.8771 \mathrm{e}-004$ \\
$(20,9,19)$ & $2.0482 \mathrm{e}-003$ & $(39,9,39)$ & $5.1461 \mathrm{e}-004$ \\
$(20,19,19)$ & $2.06074 \mathrm{e}-003$ & $(39,19,29)$ & $5.35984 \mathrm{e}-004$ \\
$(20,29,39)$ & $1.99772 \mathrm{e}-003$ & $(39,29,19)$ & $5.9185 \mathrm{e}-004$ \\
$(20,39,9)$ & $2.35229 \mathrm{e}-003$ & $(39,39,9)$ & $9.27532 \mathrm{e}-004$ \\
$(20,39,39)$ & $1.99854 \mathrm{e}-003$ & $(39,39,39)$ & $5.18641 \mathrm{e}-004$ \\
\hline
\end{tabular}

Table 7. Maximum absolute error of example 7.

\begin{tabular}{cccc}
\hline$(N, P, M)$ & Max. absolute error & $(N, P, M)$ & Max. absolute error \\
\hline$(10,9,9)$ & $2.8935 \mathrm{e}-002$ & $(29,9,39)$ & $1.14219 \mathrm{e}-002$ \\
$(10,9,29)$ & $2.9406 \mathrm{e}-002$ & $(29,19,9)$ & $4.40139 \mathrm{e}-003$ \\
$(10,19,9)$ & $2.2444 \mathrm{e}-002$ & $(29,29,19)$ & $3.40452 \mathrm{e}-003$ \\
$(10,19,19)$ & $2.29089 \mathrm{e}-002$ & $(29,29,29)$ & $3.4587 \mathrm{e}-003$ \\
$(10,29,39)$ & $2.18002 \mathrm{e}-002$ & $(29,39,19)$ & $2.97592 \mathrm{e}-003$ \\
$(10,39,29)$ & $2.13515 \mathrm{e}-002$ & $(29,39,29)$ & $3.02879 \mathrm{e}-003$ \\
$(20,9,9)$ & $1.35531 \mathrm{e}-002$ & $(39,9,19)$ & $8.42863 \mathrm{e}-002$ \\
$(20,9,19)$ & $1.38225 \mathrm{e}-002$ & $(39,9,39)$ & $7.43613 \mathrm{e}-002$ \\
$(20,19,19)$ & $7.39905 \mathrm{e}-003$ & $(39,19,29)$ & $3.30954 \mathrm{e}-002$ \\
$(20,29,39)$ & $6.2994 \mathrm{e}-003$ & $(39,29,19)$ & $6.92702 \mathrm{e}-002$ \\
$(20,39,9)$ & $5.55157 \mathrm{e}-003$ & $(39,39,9)$ & $7.68463 \mathrm{e}-003$ \\
$(20,39,39)$ & $5.89069 \mathrm{e}-003$ & $(39,39,39)$ & $5.36082 \mathrm{e}-002$ \\
\hline
\end{tabular}

doing this, we saved the number of computation and computational time. The method is shown to produce good results.

\section{REFERENCES}

[1] E. H Chao, S. F. Paul, R. C. Davidson and K. S. Fine, "Direct Numerical Solution of Poisson's Equation in Cylindrical $(r, z)$ Coordinates," Princeton University, Princeton, 1997.

[2] H. Chen, Y. Su and B. D. Shizgal, "A Direct Spectral Collocation Poisson Solver in Polar and Cylindrical Coordinates," Journal of Computational Physics, Vol. 160, No. 2, 2000, pp. 453-469. http://dx.doi.org/10.1006/jcph.2000.6461

[3] I. Christopher, G. Knorr, M. Shoucri and P. Bertrand, "Solution of the Poisson Equation in an Annulus," Journal of Computational Physics, Vol. 131, No. 2, 1997, pp. 323-326. http://dx.doi.org/10.1006/jcph.1996.5598

[4] J. C. Kalita and K. K. Ray, "A Transformation-Free HOC
Scheme for Incompressible Viscous Flows Past an Impulsively Started Circular Cylinder," Journal of Computational Physics, Vol. 228, No. 14, 2009, pp. 5207-5236. http://dx.doi.org/10.1016/j.jcp.2009.04.016

[5] M. C. Lai and W. C. Wang, "Fast Direct Solvers for Poisson Equation on 2D Polar and Spherical Geometries," Numerical Methods for Partial Differential Equations, Vol. 18, No. 1, 2002, pp. 56-68.

[6] P. N. Swarztrauber and R. A. Sweet, "The Direct Solution of the Discrete Poisson Equation on a Disk," SIAM Journal on Numerical Analysis, Vol. 10, No. 5, 1973, pp. $900-$ 907.

[7] R. C. Mittal and S. Gahlaut, "High Order Finite Difference Schemes to Solve Poisson's Equation in Cylindrical Symmetry," Communications in Applied Numerical Methods, Vol. 3, 1987, pp. 457-461.

[8] R. C. Mittal and S. Gahlaut, "High-Order Finite Differences Schemes to Solve Poisson's Equation in Polar Coordinates," IMA Journal of Numerical Analysis, Vol. 11, No. 2, 1991, pp. 261-270. http://dx.doi.org/10.1093/imanum/11.2.261

[9] C. S. Tan, "Accurate Solution of three Dimensional Poisson's Equation in Cylindrical Coordinate by Expansion in Chebyshev Polynomials," Journal of Computational Physics, Vol. 59, No. 1, 1985, pp. 81-95. http://dx.doi.org/10.1016/0021-9991(85)90108-1

[10] S. R. K. Iyengar and R. Manohar, "High Order Difference Methods for Heat Equation in Polar Cylindrical Polar Cylindrical Coordinates," Journal of Computational Physics, Vol. 77, No. 2, 1988, pp. 425-438. http://dx.doi.org/10.1016/0021-9991(88)90176-3

[11] S. R. K. Iyengar and A. Goyal, "A Note on Multigrid for the Three-Dimensional Poisson Equation in Cylindrical Coordinates," Journal of Computational and Applied Mathematics, Vol. 33, No. 2, 1990, pp. 163-169. http://dx.doi.org/10.1016/0377-0427(90)90366-8

[12] M. C. Lai and J. M. Tseng, "A Formally Fourth-Order Accurate Compact Scheme for 3D Poisson Equation in Cylindrical and Spherical Coordinates," Journal of Computational and Applied Mathematics, Vol. 201, No. 1, 2007, pp. 175-181. http://dx.doi.org/10.1016/j.cam.2006.02.011

[13] J. Xu, P. N. Ostroumov and J. Nolen, "A Parallel 3D Poisson Solver for Space Charge Simulation in Cylindrical Coordinates," Computer Physics Communications, Vol. 178, No. 4, 2008, pp. 290-300. http://dx.doi.org/10.1016/j.cpc.2007.09.008

[14] M. A. Malcolm and J. Palmer, "A Fast Method for Solving a Class of Tri-Diagonal Linear Systems," Communications of the ACM, Vol. 17, No. 1, 1974, pp. 14-17. http://dx.doi.org/10.1145/360767.360777

[15] R. W. Hockney, "A Fast Direct Solution of Poisson Equation Using Fourier Analysis," JACM, Vol. 12, No. 1, 1965, pp. 95-113. http://dx.doi.org/10.1145/321250.321259 